\title{
A case of neglect...
}

$\int$ ooking at the magazines of member societies of EPS and at Europhysics News, we have to conclude that they all have suffered a stroke. The $10 \%$ of the physics community that is university-employed and thrives on pure research is well served. Most of the content is geared to serve this minority. The other $90 \%$, earning their daily wages in the 'real world', is neglected.

In medicine, 'neglect' is a well-defined term relating to cerebral damage due to an infarct. A patient with left-handed neglect will only eat what is served on the right-hand side of his plate. The damage does not heal. Is this the case for the physical societies? Or is there hope for healing?

\section{Real-world careers}

The majority of careers for physicists are not in academia, but elsewhere. Think of industry, consultancy, teaching, option trading, policy-making, think tanks and all the other institutions that serve society and keep our economy going. Some of these physicists just stop reading the bulletins but hold on to their membership. This can be considered as charity of disgruntled professionals for the academic membership. However, most will discontinue their membership and switch to other magazines.

For students at the M.Sc. and $\mathrm{PhD}$ level this narrow outlook on their professional life is rather

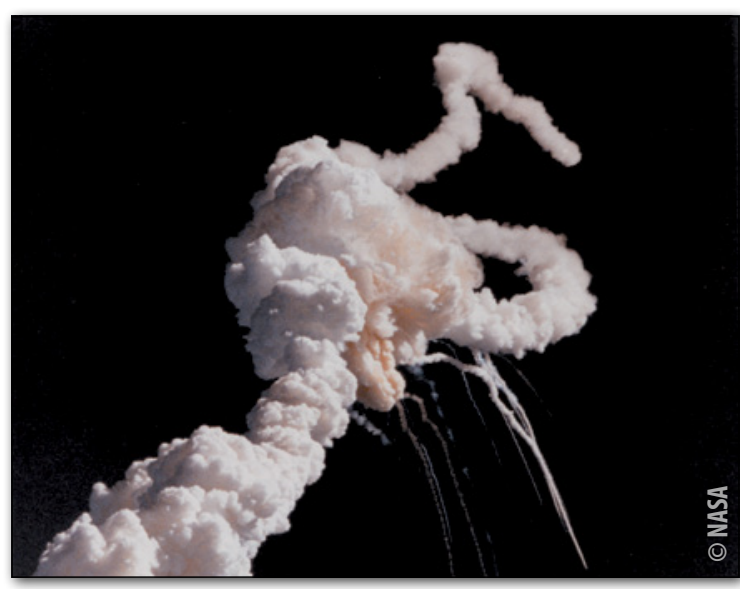

forgotten that a university should be the major supplier of well-trained talent to society? Top level research is an excellent training tool, but it comes second to the real output of academia: independent, out-of-the-box thinking, talented youngsters, well trained in abstract analysis and a scientific approach to solve new problems in real life.

\section{Community building}

Wouldn't it be wonderful to cater to all physicists with a news bulletin that reflects the broad background of typical careers? Wouldn't it be exciting to increase our membership five-fold when all the 'lost sheep' return to the fold, by a bulletin that revitalizes all of us, including academia? Think of the financial basis this would supply for EPS. Think of the impact this will have when talking to the EU.

As an example, look at the Dutch Physical Society (NNV). In 2008, the membership was 3500 including retired members. With a B.Sc. at age 22, a lifespan of 85 years - for a male with an academic degree - and an estimated output of 250 students graduating each year from a Dutch university, one obtains the incredible number of $63 \times 250=$ 15,750 potential members. The numbers will not differ that much for other member societies of EPS. This implies that we can gain a factor of FOUR in membership if we really shift our focus from the confusing. Much worse is that university students are discouraged to become a member at all: they are already lost before the inaugural ball!

Why is this happening? Is it due to partial blindness of our university professors, who forget to tell about the beautiful and interesting work that physicists do in their careers outside university? Is it just self-interest, to fill the $\mathrm{PhD}$ positions in their research groups? Have we minority in academia to the majority elsewhere.

A huge challenge lies before us! Of course, we cannot change overnight. But we can shift the focus of Europhysics News into a direction that reflects the majority of our community. We can make a difference and start nurturing the interaction between academia and industry. Academia should realize its role is to serve and not to dominate. 